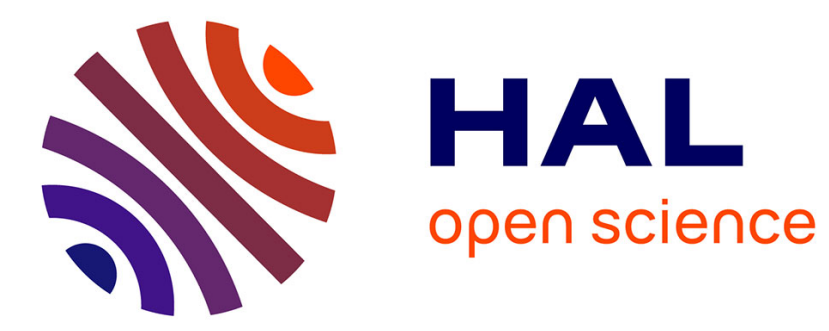

\title{
LPV state-feedback controller for Attitude/Altitude stabilization of a mass-varying quadcopter
}

The Hung Pham, Dalil Ichalal, Saïd Mammar

\section{To cite this version:}

The Hung Pham, Dalil Ichalal, Saïd Mammar. LPV state-feedback controller for Attitude/Altitude stabilization of a mass-varying quadcopter. 20th International Conference on Control, Automation and Systems (ICCAS 2020), Oct 2020, Busan, South Korea. pp.212-218, 10.23919/ICCAS50221.2020.9268310 . hal-03092141

\section{HAL Id: hal-03092141 \\ https://hal.science/hal-03092141}

Submitted on 2 Mar 2021

HAL is a multi-disciplinary open access archive for the deposit and dissemination of scientific research documents, whether they are published or not. The documents may come from teaching and research institutions in France or abroad, or from public or private research centers.
L'archive ouverte pluridisciplinaire HAL, est destinée au dépôt et à la diffusion de documents scientifiques de niveau recherche, publiés ou non, émanant des établissements d'enseignement et de recherche français ou étrangers, des laboratoires publics ou privés. 


\title{
LPV state-feedback controller for Attitude/Altitude stabilization of a mass-varying quadcopter
}

\author{
The Hung PHAM ${ }^{1 *}$ and Dalil ICHALAL ${ }^{2}$ and Said MAMMAR ${ }^{3}$ \\ 1,2,3 IBISC, Evry-Val-d'Essonne University, Universite Paris-Saclay, Evry, France(thehung.pham@ univ-evry.fr, \\ dalil.ichalal@univ-evry.fr, said.mammar@univ-evry.fr) \\ ${ }^{1}$ Le Quy Don Technical University, Hanoi, Viet Nam (thehungpham83@ gmail.com) * Corresponding author
}

\begin{abstract}
This paper concerns the problem of attitude/altitude control of a quadrotor. The main contribution consists of developing a simple Linear Parameter Varying (LPV) model which includes the motor dynamics, weight, and moment of inertia variations. In addition, a robust LPV $H_{\infty}$ state-feedback controller is proposed. It allows us to perform both a reference trajectory tracking and disturbance rejection for attitude/altitude control of a mass-varying quadcopter. First, an augmented state which includes the integration of the trajectory errors for improving tracking control is computed. Next, to penalize the control inputs of the attitude/altitude system, weight functions are also added to the previous augmented system. Then, an $H_{\infty}$ state-feedback controller is designed by solving a set of Linear Matrix Inequalities (LMI) obtained from the Bounded Real Lemma and LMI region characterization. Simulations are conducted for several types of disturbances (sine, impulse, step, and random) and variations (slow and abrupt) of mass and moments of inertia. The reference path (sine, impulse, and step) is well-followed showing the ability of the design method to handle different performance objectives.
\end{abstract}

Keywords: Quadcopter, Linear Parameter Varying (LPV), Takagi-Sugeno (TS), Linear Matrix Inequality (LMI), Statefeedback controller.

\section{INTRODUCTION}

Unmanned aerial vehicles are a really important device used to perform predefined or autonomous tasks in a dangerous and/or inaccessible environment, such as industrial inspection of solar parks, wind parks, power lines, engines and plants, and industrial parks, bridge inspection, visual structure assessment and monitoring, inspection and survey of structures, etc. Thanks to their wide applicability, they have been seriously researched and developed. To correctly perform the assigned tasks, it is required to design the vertical takeoff and landing (VTOL)UAV which is highly maneuverable and extremely stable. To perform the movement in space, the position of the UAV is mostly manually controlled by an operator through a remote-control system using visual feedback from an on-board camera, during which time the UAV's attitude/altitude is automatically stabilized by an on-board controller.

Attitude/Altitude controller design is an important task since it equips the UAV with the ability to maintain the desired orientation, altitude, and to prevent the vehicle from flipping over and colliding with the surrounding environment when the pilot performs the desired maneuvers. The attitude control problem for UAV has been studied by numerous researchers and several controllers have been proposed and applied. In [1], a robust adaptive tracking controller for the attitude of a quadcopter is presented by designing an adaptive law to estimate the inertia matrix of the vehicle. The algorithm can also be extended to a general class of unstructured disturbances. In [2][3][4] a robust nonlinear controller is applied by a combination of backstepping technique and sliding mode control methods to enhance the tracking performance of the attitude and position of a quadrotor UAV under bounded uncertainties and time-varying perturbations. In [5], the problem of attitude and altitude control was addressed. An error model simplifies the problem and a LPV controller has been proposed under the form of a state feedback.

The mass and moments of inertia of UAVs are important constraints to take into account in applications for spraying pesticides, the mass and moments of inertia of flying equipment may vary slowly over time. Meanwhile, in goods transportation, the mass and moments of inertia of aircraft may/often/typically change abruptly. Mass variation implies changes in moments of inertia. Because of the changes of the quadcopter's parameter (mass and moments of inertia), the dynamic model of the quadcopter is also varying.

The aim of this paper is to propose a simple design procedure of LPV state feedback for the attitude/altitude stabilization problem of the mass-varying quadrotor aircraft. In our context, the main objective of the state feedback synthesis is to handle the mass, moments of inertia and rotors velocity variations which are assumed to be measured. This is achieved using a LPV formalism which allows to obtain a Takagi-Sugeno model with sixteen sub-models depending on the extremal values of the varying parameters. The controller is then synthesized on the basis of a sixteen-sub-models system.

The remainder of the paper is organized as follows: Section 2 presents the dynamical model of the quadcopter and some preliminary concepts for designing the objective of the multi-objective controller. Section 3 is dedicated for designing the LPV state feedback controller for the attitude/altitude of the quadcopter. The controller is practically synthesized in section 4 , while simulation results are presented in section 5. Finally conclusions and 
some future work proposals wrap up the paper.

Notation: The notations in this paper are fairly standard. The notation $X \succ 0(X \prec 0)$ where $X$ is symmetric matrix, denotes that $X$ is positive (negative) definite. $H e[X]$ is hermitian operator defined as $H e[X]:=X+X^{T}$. The symbol $(*)^{T}$ generically denotes each of its symmetric blocks. The $\mathrm{N}$-unit simplex, denoted by $\Lambda_{N}$, is defined as the set $\Lambda_{N}=\left\{\chi \in R_{\geq 0}^{N}: \sum_{i=1}^{N} \chi_{i}=1\right\}$. The set of positive definite matrix is denoted as $S_{\succ 0}^{n}$.

\section{SYSTEM MODEL AND PROBLEM STATEMENT}

This paper aims to develop a state feedback controller for a quadcopter. The vehicle has six degrees of freedom for a quadcopter and only four actuators. It is thus underactuated.

\subsection{Quadrotor model}

A quadcopter is a helicopter which consists of a rigid cross frame equipped with four rotors as shown in Fig. 1. Its four rotors generate four independent thrusts. In order to avoid the yaw drift due to the reactive torques, the quadrotor aircraft is configured such that the set of rotors $M_{2}, M_{4}$ (left-right) revolve clockwise $(\mathrm{CW})$ at angular speeds $\omega_{2}$ and $\omega_{4}$, respectively generating thrusts of $\tau_{2}$ and $\tau_{4}$, while the pair of rotors $M_{1}, M_{3}$ (frontrear) rotates at angular speeds $\omega_{1}$ and $\omega_{3}$ in counterclockwise $(\mathrm{CCW})$ direction generating thrusts of $\tau_{1}$ and $\tau_{3}$. The direction of rotation of the rotors are fixed (i.e., $\left.\omega_{i} \geq 0, i \in\{1,2,3,4\}\right)$. The forward/backward, left/right and the yaw motions are achieved through a differential control strategy of the thrust generated by each rotor.

If a yaw motion is desired, the thrust of one set of rotors has to be reduced and the thrust of the other set are increased while maintaining the same total thrust to avoid an up (down) motion. Therefore, the yaw motion is then realized in the direction of the induced reactive torque. Besides, forward (backward) motion is achieved by pitching in the desired direction by increasing the rear (front) rotor thrust and decreasing the front (rear) rotor thrust to maintain the total thrust. Finally, a sideways motion is achieved by rolling in the desired direction by increasing the left (right) rotor thrust and decreasing the right (left) rotor thrust to maintain the total thrust.

Let $I=\left\{e_{x}, e_{y}, e_{z}\right\}$ denotes an inertial frame, and $A=$ $\left\{e_{1}, e_{2}, e_{3}\right\}$ denotes a frame rigidly attached to the aircraft as shown in Fig. 1.

The mathematical model of the quadcopter was generated by the techniques of both Euler-Newton [6] and Euler-Lagrange [7], given as follows:

$$
\left\{\begin{aligned}
\ddot{x}_{c} & =(\sin \psi \sin \varphi+\cos \psi \sin \theta \cos \varphi) \frac{U_{1}}{m} \\
\ddot{y}_{c} & =(\sin \psi \sin \theta \cos \varphi-\cos \psi \sin \varphi) \frac{U_{1}}{m} \\
\ddot{z}_{c} & =(\cos \theta \cos \varphi) \frac{U_{1}}{m}-g \\
\ddot{\varphi} & =\frac{I_{y}-I_{z}}{I_{x}} \dot{\theta} \dot{\psi}-\frac{J_{r} \Omega_{r}}{I_{x}} \dot{\theta}+\frac{l}{I_{x}} U_{2} \\
\ddot{\theta} & =\frac{I_{z}-I_{x}}{I_{y}} \dot{\varphi} \dot{\psi}+\frac{J_{r} \Omega_{r}}{I_{y}} \dot{\varphi}+\frac{l}{I_{y}} U_{3} \\
\ddot{\psi} & =\frac{I_{x}-I_{y}}{I_{z}} \dot{\varphi} \dot{\theta}+\frac{1}{I_{z}} U_{4}
\end{aligned}\right.
$$

where $m$ denotes the mass the of the quadcopter, $\left(x_{c}, y_{c}, z_{c}\right)$ are the three positions of the center of mass, $(\varphi, \theta, \psi)$ are the three Euler angles, $I_{x}, I_{y}$, and $I_{z}$ are the moments of inertia w.r.t the three axis $x, y$, and $z$ respectively; $J_{r}$ is the moment of inertia of the propellers, $l$ represents the distance from the rotors to the center of mass of the quadrotor aircraft. $\Omega_{r}$ is the overall residual propeller angular speed. The quadcopter's inputs are: the thrust force $\left(U_{1}\right)$, three torques (roll torque $\left(U_{2}\right)$, pitch torque $\left(U_{3}\right)$, and yaw torque $\left.\left(U_{4}\right)\right)$. The force and torques are related on the rotor speed as follows:

$$
\left\{\begin{aligned}
U_{1} & =k_{f}\left(\omega_{1}^{2}+\omega_{2}^{2}+\omega_{3}^{2}+\omega_{4}^{2}\right)=\sum_{i=1}^{4} T_{i} \\
U_{2} & =k_{f}\left(\omega_{4}^{2}-\omega_{2}^{2}\right)=T_{4}-T_{2} \\
U_{3} & =k_{f}\left(\omega_{3}^{2}-\omega_{1}^{2}\right)=T_{3}-T_{1} \\
U_{4} & =k_{z}\left(-\omega_{1}^{2}+\omega_{2}^{2}-\omega_{3}^{2}+\omega_{4}^{2}\right) \\
& =\left(T_{2}+T_{4}\right)-\left(T_{1}+T_{3}\right)
\end{aligned}\right.
$$

and

$\Omega_{r}=\omega_{1}-\omega_{2}+\omega_{3}-\omega_{4}$

where $\omega_{i}$ for $i=1,2,3,4$ denotes the $i$-th rotor velocity, and $T_{i}$ for $i=1,2,3,4$ are the thrust generated by the $i$-th rotor and the thrust $T_{i}(t)$ is a function of the rotor speed defined by

$T_{i}(t)=k_{f} \omega_{i}^{2}$

where $k_{f}$ is the constant coefficient.

The first three equations of the system differential equations in (1) denote the translational movement, while the last three present the rotational movement of the quadcopter. We restrict the purpose of the paper to the attitude tracking. Thus, the equations related to the longitudinal and lateral motions of the quadcopter in (1) are removed.

\subsection{Actuator model}

Adopting an actuator model has twofold. First of them is to reflect the low pass filtering of each actuator with a time constant $\kappa_{i}, i=1,2,3,4$. The second allows us to prevent the $B$ matrix of the obtained state-space representation to be parameter dependent. Each actuator thrust Laplace transform is given by

$\mathscr{T}_{i}(s)=\frac{K_{i}}{1+\kappa_{i} s} V_{i}(s), \quad i=1,2,3,4$

$\mathscr{T}_{i}$ is the Laplace transform of the thrust $T_{i}(t), V_{i}$ is the pulse width modulation (PWM) voltage applied to rotor $i$, and $K_{i}$ is the armature gain.

The corresponding differential equation is

$\dot{T}_{i}=-\frac{1}{\kappa_{i}} T_{i}+\frac{K_{i}}{\kappa_{i}} V_{i}$

Remark 1: Based on the PWM applied to each rotor, the rotor speed $\omega_{i}$ can be estimated. Thus, the residual speed $\Omega_{r}=\omega_{2}+\omega_{4}-\omega_{1}-\omega_{3}$ can also be estimated. 


\subsection{Simplified model}

The previous model still exhibits too many parameters and its polytopic representation will involve at least $2^{6}$ sub-models. If one considers control synthesis using LMI methods, the solvability of the resulting LMI conditions in this case is quite compromised due to conservativeness of conditions which will request the common stabilization of a huge number of sub-models. In order to reduce this number, we adopt here a simplified model. In particular, suppose that $I_{x}=I_{y}$ and $\varphi$ and $\theta$ are small so that $\cos \varphi \cos \theta \approx 1$.

We also assume that the attitude/altitude subsystem of the quadcopter is affected by torques $d_{\varphi}, d_{\theta}, d_{\psi}$, and force $d_{z}$ allowing to write:

$$
\left\{\begin{aligned}
\ddot{\varphi} & =-\frac{J_{r} \Omega_{r}}{I_{x}} \dot{\theta}+\frac{l}{I_{x}}\left(T_{4}-T_{2}\right)+\frac{1}{I_{x}} d_{\varphi} \\
\ddot{\theta} & =\frac{J_{r} \Omega_{r}}{I_{x}} \dot{\varphi}+\frac{l}{I_{x}}\left(T_{3}-T_{1}\right)+\frac{1}{I_{x}} d_{\theta} \\
\ddot{\psi} & =\frac{\left(T_{4}+T_{2}\right)-\left(T_{3}+T_{1}\right)}{I_{I_{x}}}+\frac{1}{I_{z}} d_{\psi} \\
\ddot{z} & =\frac{T_{1}+T_{2}+T_{3}+T_{4}}{m}+\frac{1}{m} d_{z}-g
\end{aligned}\right.
$$

Note that $g$ is also the disturbance to the system. Then the disturbances can be written in the vector form as $d(t)=$ $\left[\begin{array}{lllll}d_{\varphi} & d_{\theta} & d_{\psi} & d_{z} & g\end{array}\right]^{T}$.

Then, the LPV model of attitude/altitude sub-system in (1) is performed as:

$$
\begin{aligned}
& \dot{x}(t)=A\left(I_{x}, I_{z}, \Omega_{r}, m\right) x(t)+B\left(I_{x}, I_{z}, \Omega_{r}, m\right) u(t) \\
& \quad+E\left(I_{x}, I_{z}, \Omega_{r}, m\right) d(t)
\end{aligned}
$$

where state $x=\left(\varphi, \theta, \psi, z_{c}, \dot{\varphi}, \dot{\theta}, \dot{\psi}, \dot{z}_{c}, T_{1}, T_{2}, T_{3}, T_{4}\right)^{T}$ has twelve components, the control input vector is composed of the four motor voltages $u=\left(v_{1}, v_{2}, v_{3}, v_{4}\right)^{T}$, and the system matrices $A\left(I_{x}, I_{z}, \Omega_{r}, m\right), B\left(I_{x}, I_{z}, \Omega_{r}, m\right)$, and $E\left(I_{x}, I_{z}, \Omega_{r}, m\right)$ are

$$
\begin{aligned}
& A\left(I_{x}, I_{z}, \Omega_{r}, m\right)=\left[\begin{array}{lll}
A_{11} & A_{12} & A_{13}
\end{array}\right] \\
& B\left(I_{x}, I_{z}, \Omega_{r}, m\right)=\left[\begin{array}{l}
0_{9 \times 3} \\
B_{*}
\end{array}\right] \\
& E\left(I_{x}, I_{z}, \Omega_{r}, m\right)=\left[\begin{array}{c}
0_{4 \times 5} \\
E_{*} \\
0_{4 \times 5}
\end{array}\right]
\end{aligned}
$$

where

$A_{11}=\left[0_{12 \times 4}\right]$

$$
A_{12}=\left[\begin{array}{cccc}
1 & 0 & 0 & 0 \\
0 & 1 & 0 & 0 \\
0 & 0 & 1 & 0 \\
0 & 0 & 0 & 1 \\
0 & \frac{I_{x}-I_{z}}{I_{x}} \dot{\psi}-\frac{J_{r}}{I_{x}} \Omega_{r} & 0 & 0 \\
-\frac{I_{x}-I_{z}}{I_{x}} \dot{\psi}+\frac{J_{r}}{I_{x}} \Omega_{r} & 0 & 0 & 0 \\
0 & 0 & 0 & 0 \\
0 & 0 & 0 & 0 \\
0 & 0 & 0 & 0 \\
0 & 0 & 0 & 0 \\
0 & 0 & 0 & 0 \\
0 & 0 & 0 & 0
\end{array}\right]
$$

$$
\begin{aligned}
& A_{13}= {\left[\begin{array}{cccc}
0 & 0 & 0 & 0 \\
0 & 0 & 0 & 0 \\
0 & 0 & 0 & 0 \\
0 & 0 & 0 & 0 \\
0 & -l / I_{x} & 0 & l / I_{x} \\
-l / I_{x} & 0 & l / I_{x} & 0 \\
-l / I_{z} & l / I_{z} & -l / I_{z} & l / I_{z} \\
1 / m & 1 / m & 1 / m & 1 / m \\
-1 / \kappa_{1} & 0 & 0 & 0 \\
0 & -1 / \kappa_{2} & 0 & 0 \\
0 & 0 & -1 / \kappa_{3} & 0 \\
0 & 0 & 0 & -1 / \kappa_{4}
\end{array}\right] } \\
& B_{*}= {\left[\begin{array}{cccc}
K_{1} / \kappa_{1} & 0 & 0 & 0 \\
0 & K_{2} / \kappa_{2} & 0 & 0 \\
0 & 0 & K_{3} / \kappa_{3} & 0 \\
0 & 0 & 0 & K_{4} / \kappa_{4}
\end{array}\right] } \\
& E_{*}= \\
& {\left[\begin{array}{ccccc}
1 / I_{x} & 0 & 0 & 0 & 0 \\
0 & 1 / I_{x} & 0 & 0 & 0 \\
0 & 0 & 1 / I_{z} & 0 & 0 \\
0 & 0 & 0 & 1 / m & -1
\end{array}\right] }
\end{aligned}
$$

Note that, as described above, the system matrix $B$ is time invariant.

The main objective of the control design procedure is to synthesize a state feedback controller that could be scheduled according to mass, moment of inertias and rotor speeds variations. As it can be seen from the simplified quadrotor model, it is linear in all the parameters. One can thus obtain a LPV model depending on four parameters, the moment of inertia with respect to x axis $I_{x} \in$ $\left[\begin{array}{ll}I_{x} & \overline{I_{x}}\end{array}\right]$, the moment of inertia with respect to $z$ axis $I_{z} \in\left[\begin{array}{cc}I_{z} & \overline{I_{z}}\end{array}\right]$, the residual velocity $\Omega_{r} \in\left[\begin{array}{cc}\Omega_{r} & \overline{\Omega_{r}}\end{array}\right]$, and the mass $m \in\left[\begin{array}{ll}\frac{m}{m} & \bar{m}\end{array}\right]$ of the quadcopter.

Thus a Takagi-Sugeno (TS) model with sixteen submodels could be obtained depending on the extremal values of the parameters. This representation is called nonlinear sector approximation [8]. In fact, if we define the varying parameters as follows

$$
\begin{aligned}
& \rho_{1}=\frac{1}{I_{x}} \in\left[\begin{array}{cc}
\frac{1}{\overline{I_{x}}} & \frac{1}{I_{x}}
\end{array}\right]=\left[\begin{array}{ll}
\rho_{1} & \bar{\rho}_{1}
\end{array}\right] \\
& \rho_{2}=\frac{1}{I_{z}} \in\left[\begin{array}{ll}
\frac{1}{\overline{I_{z}}} & \frac{1}{I_{z}}
\end{array}\right]=\left[\begin{array}{ll}
\underline{\rho}_{2} & \overline{\rho_{2}}
\end{array}\right] \\
& \rho_{3}=\frac{1}{m} \in\left[\begin{array}{cc}
\frac{1}{\bar{m}} & \frac{1}{\bar{m}}
\end{array}\right]=\left[\begin{array}{ll}
\underline{\rho}_{3} & \bar{\rho}_{3}
\end{array}\right] \\
& \rho_{4}=\frac{I_{x}-I_{z}}{I_{x}} \dot{\psi}-\frac{J_{r}}{I_{x}} \Omega_{r} \in\left[\begin{array}{ll}
\underline{\rho}_{4} & \bar{\rho}_{4}
\end{array}\right]
\end{aligned}
$$

then a sixteen sub-models TS system is achieved

$\dot{x}(t)=\sum_{i=1}^{16} \mu_{i}\left(\bar{A}_{i} x(t)+B u(t)+\bar{E}_{i} d(t)\right)$

where $\mu_{i} \geq 0,1 \leq i \leq 16, \sum_{1}^{16} \mu_{i}=1$ and

$$
\begin{aligned}
& \mu_{1}(\rho(t))=m_{11} m_{21} m_{31} m_{41} ; \mu_{2}(\rho(t))=m_{11} m_{21} m_{31} m_{42} \\
& \mu_{3}(\rho(t))=m_{11} m_{21} m_{32} m_{41} ; \mu_{4}(\rho(t))=m_{11} m_{21} m_{32} m_{42} \\
& \mu_{5}(\rho(t))=m_{11} m_{22} m_{31} m_{41} ; \mu_{6}(\rho(t))=m_{11} m_{22} m_{31} m_{42} \\
& \mu_{7}(\rho(t))=m_{11} m_{22} m_{32} m_{41} ; \mu_{8}(\rho(t))=m_{11} m_{22} m_{32} m_{42} \\
& \mu_{9}(\rho(t))=m_{12} m_{21} m_{31} m_{41} ; \mu_{10}(\rho(t))=m_{12} m_{21} m_{31} m_{42} \\
& \mu_{11}(\rho(t))=m_{12} m_{21} m_{32} m_{41} ; \mu_{12}(\rho(t))=m_{12} m_{21} m_{32} m_{42} \\
& \mu_{13}(\rho(t))=m_{12} m_{22} m_{31} m_{41} ; \mu_{14}(\rho(t))=m_{12} m_{22} m_{31} m_{42} \\
& \mu_{15}(\rho(t))=m_{12} m_{22} m_{32} m_{41} ; \mu_{16}(\rho(t))=m_{12} m_{22} m_{32} m_{42}
\end{aligned}
$$


with

$$
\begin{array}{ll}
m_{11}(\rho(t))=\frac{\bar{\rho}_{1}-\rho_{1}}{\bar{\rho}_{1}-\rho_{1}}, & m_{12}(\rho(t))=1-m_{11} \\
m_{21}(\rho(t))=\frac{\bar{\rho}_{2}-\bar{\rho}_{2}}{\bar{\rho}_{2}-\rho_{2}}, & m_{22}(\rho(t))=1-m_{21} \\
m_{31}(\rho(t))=\frac{\bar{\rho}_{3}-\bar{\rho}_{3}}{\bar{\rho}_{3}-\bar{\rho}_{3}}, & m_{32}(\rho(t))=1-m_{31} \\
m_{41}(\rho(t))=\frac{\bar{\rho}_{4}-\bar{\rho}_{4}}{\bar{\rho}_{4}-\underline{\rho}_{4}}, & m_{42}(\rho(t))=1-m_{41}
\end{array}
$$

The matrices $\bar{A}_{i}, 1 \leq i \leq 16$ are obtained from

$$
\begin{aligned}
& \bar{A}_{1}=A\left(\underline{\rho}_{1}, \underline{\rho}_{2}, \underline{\rho}_{3}, \underline{\rho}_{4}\right) ; \bar{A}_{2}=A\left(\underline{\rho}_{1}, \underline{\rho}_{2}, \underline{\rho}_{3}, \bar{\rho}_{4}\right) \\
& \bar{A}_{3}=A\left(\underline{\rho}_{1}, \underline{\rho}_{2}, \bar{\rho}_{3}, \underline{\rho}_{4}\right) ; \bar{A}_{4}=A\left(\underline{\rho}_{1}, \underline{\rho}_{2}, \bar{\rho}_{3}, \bar{\rho}_{4}\right) \\
& \bar{A}_{5}=A\left(\underline{\rho}_{1}, \bar{\rho}_{2}, \underline{\rho}_{3}, \underline{\rho}_{4}\right) ; \bar{A}_{6}=A\left(\underline{\rho}_{1}, \bar{\rho}_{2}, \underline{\rho}_{3}, \bar{\rho}_{4}\right) \\
& \bar{A}_{7}=A\left(\underline{\rho}_{1}, \bar{\rho}_{2}, \bar{\rho}_{3}, \underline{\rho}_{4}\right) ; \bar{A}_{8}=A\left(\underline{\rho}_{1}, \bar{\rho}_{2}, \bar{\rho}_{3}, \bar{\rho}_{4}\right) \\
& \bar{A}_{9}=A\left(\bar{\rho}_{1}, \underline{\rho}_{2}, \underline{\rho}_{3}, \underline{\rho}_{4}\right) ; \bar{A}_{10}=A\left(\bar{\rho}_{1}, \underline{\rho}_{2}, \underline{\rho}_{3}, \bar{\rho}_{4}\right) \\
& \bar{A}_{11}=A\left(\bar{\rho}_{1}, \underline{\rho}_{2}, \bar{\rho}_{3}, \underline{\rho}_{4}\right) ; \bar{A}_{12}=A\left(\bar{\rho}_{1}, \underline{\rho}_{2}, \bar{\rho}_{3}, \bar{\rho}_{4}\right) \\
& \bar{A}_{13}=A\left(\bar{\rho}_{1}, \bar{\rho}_{2}, \underline{\rho}_{3}, \underline{\rho}_{4}\right) ; \bar{A}_{14}=A\left(\bar{\rho}_{1}, \bar{\rho}_{2}, \underline{\rho}_{3}, \bar{\rho}_{4}\right) \\
& \bar{A}_{15}=A\left(\bar{\rho}_{1}, \bar{\rho}_{2}, \bar{\rho}_{3}, \underline{\rho}_{4}\right) ; \bar{A}_{16}=A\left(\bar{\rho}_{1}, \bar{\rho}_{2}, \bar{\rho}_{3}, \bar{\rho}_{4}\right)
\end{aligned}
$$

The output vector $y=\left[\begin{array}{cccc}\varphi & \theta & \psi & z\end{array}\right]^{T}$ is constituted by the quadrotor attitude/altitude position which are obtained from

$y=C x+D u$

where $C=\left[\begin{array}{ll}I_{4 \times 4} & 0_{4 \times 8}\end{array}\right]$ and $D=\left[0_{4 \times 4}\right]$

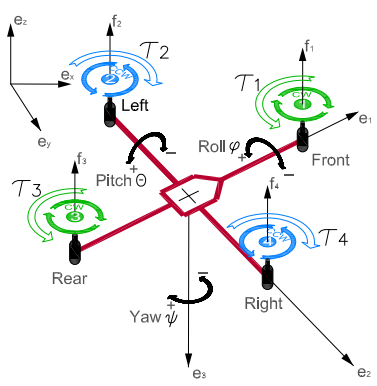

Fig. 1 Quadcopter

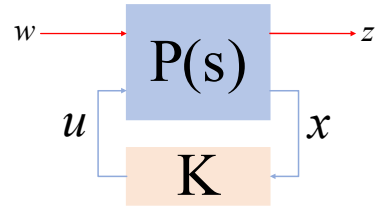

Fig. 2 Control structure
Remark 2: Suppose the quadcopter is attached with $n$ objects $o_{1}, \ldots, o_{n}$, and the mass of quadcopter and objects are $m_{q}, m_{o_{1}}, \ldots, m_{o_{n}}$ respectively. Therefore, the mass of the system consists of the quadcopter and $n$ objects can be easily calculated by the equation $m=m_{q}+m_{o_{1}}+\ldots+$ $m_{o_{n}}$. When $o_{i}$ - the $i$-th object is detached from the quadcopter for $i=n, \ldots, 1$, the remaining mass of the system can be recalculated.

Depending on the mass and shape of each object, ones can calculate its moments of inertia around the axes passing through its center of mass. When attaching these objects to the quadcopter, based on their shapes and positions with respect to the center of gravity $G$ of the quadcopter, their the moments of inertia with respect to the three axes $I_{x}, I_{y}, I_{z}$ of the quadcopter can be calculated. Thus the moment of inertia of the system which contains quadcopter and $n$ objects $o_{1}, \ldots, o_{n}$ relative to $I_{x}, I_{y}, I_{z}$ can be calculated.
Another online approach to estimate the geometric and inertia parameters of a multirotor aerial vehicle is already developed in [9].

Remark 3: From Remarks 1 and 2 one can see that all the varying parameters can be estimated in real time.

\subsection{Preliminary concepts}

Suppose the polytopic LPV system is of the form

$$
\begin{aligned}
& \dot{x}(t)=A(\rho(t)) x(t)+B_{1}(\rho(t)) w(t)+B_{2} u(t) \\
& z(t)=C(\rho(t)) x(t)+D_{11}(t) w(t)+D_{12} u(t) \\
& x(0)=x_{0}
\end{aligned}
$$

where $x \in R^{n}$ is the system state, $u \in R^{m}$ is the control input, $w \in R^{p}$ is the exogenous input, and $z \in R^{q}$ is the controlled output. The $\rho$-parameter dependent system matrices is defined as

$$
\begin{aligned}
& A(\rho(t))=\sum_{i=1}^{N} \mu_{i}(\rho(t)) A_{i} ; \quad B_{1}(\rho(t))=\sum_{i=1}^{N} \mu_{i}(\rho(t)) B_{1_{i}} \\
& C_{1}(\rho(t))=\sum_{i=1}^{N} \mu_{i}(\rho(t)) C_{1_{i}} ; D_{11}(\rho(t))=\sum_{i=1}^{N} \mu_{i}(\rho(t)) D_{11_{i}}
\end{aligned}
$$

while $B_{2}$ and $D_{12}$ are constant matrices.

The purpose of this section is to design a LPV statefeedback control law

$u(t)=\sum_{i=1}^{N} K_{i} x(t)$

such that:

- The $H_{\infty}$ norm of the system (13) from $w$ to $z$ (as depicted in Fig. 2) is guaranteed to be smaller than some predefined value $\gamma>0$ for tracking and disturbance rejection (robustness). This condition is guaranteed by the following Theorem 1 below

- Closed loop poles are placed in a predefined LMI region [11] for ensuring the ability of fast and well-damped transient response. The closed-loop poles satisfy the condition $\operatorname{Re}\left(e i g\left(A+B_{2} Y X^{-1}\right)\right)<-\alpha$ for $\alpha>0$

$$
\begin{aligned}
& \operatorname{Re}\left(\operatorname{eig}\left(A+B_{2} Y X^{-1}\right)\right)<-\alpha, \quad \alpha>0 \Leftrightarrow \\
& \exists X=X^{T} \succ 0 \\
& \text { s.t } 2 \alpha X+\mathrm{He}\left(A_{i} X+B_{2} Y_{i}\right) \prec 0, i=1, \ldots, N
\end{aligned}
$$

Theorem 1: (Theorem 3.4.1 in [10]) The LPV system (13) is quadratically stabilizable using a statefeedback of the form (14) if there exist a matrix $X \in S_{\succ 0}^{n}$, matrices $Y_{i} \in^{m \times n}, i=1, \ldots, N$, and a scalar $\gamma>0$ such that the LMIs

$$
\left[\begin{array}{ccc}
H e\left(A_{i} X+B_{2} Y_{i}\right) & (*)^{T} & (*)^{T} \\
E_{i}^{T} & -\gamma I_{p} & (*)^{T} \\
C_{i} X+D_{12} Y_{i} & D_{11_{i}} & -\gamma I_{q}
\end{array}\right] \prec 0
$$

hold for all $i=1, \ldots, N$. Moreover, the state-feedback control law given by (14) with the matrices $K_{i}=Y_{i} X^{-1}$ ensures that the $L_{2}$-gain of the transfer $w \rightarrow z$ is smaller than $\gamma>0$ for all $\mu: R_{\geq 0} \rightarrow \Lambda_{N}$.

Then the state-feedback control law given by (14) with the matrices $K_{i}=Y_{i} X^{-1}$ satisfy Theorem 1 and equation (15) ensures that the $L_{2}$-gain of the transfer $w \rightarrow z$ is smaller than $\gamma>0$ for all $\mu: R_{\geq 0} \rightarrow \Lambda_{N}$ and the poles of the close loop system satisfy condition $\operatorname{Re}\left(\operatorname{eig}\left(A+B_{2} Y X^{-1}\right)\right)<-\alpha, \quad \alpha>0$. 


\section{LPV ATTITUDE STATE FEEDBACK CONTROLLER DESIGN}

In this section, we aim to design a $H_{\infty}$ LPV feedback control scheme for the attitude/altitude stabilization of the quadrotor aircraft.

First, the output $y=\left[\begin{array}{cccc}\varphi & \theta & \psi & z_{c}\end{array}\right]^{T}$ of the system must track $r=\left[\begin{array}{cccc}\varphi_{\text {ref }} & \theta_{\text {ref }} & \psi_{\text {ref }} & z_{\text {ref }}\end{array}\right]^{T}$, a reference trajectory. Therefore, to achieve these objectives, the outputs of the integrator are considered as extra state variables $x_{e}=\left[\begin{array}{llll}x_{\varphi} & x_{\theta} & x_{\psi} & x_{z}\end{array}\right]^{T}$ as

$$
\begin{aligned}
& x_{\varphi}=\int_{0}^{t} e_{\varphi}(\delta) d \delta, \quad e_{\varphi}=\varphi_{r e f}-\varphi \\
& x_{\theta}=\int_{0}^{t} e_{\theta}(\delta) d \delta, \quad e_{\theta}=\theta_{r e f}-\theta \\
& x_{\psi}=\int_{0}^{t} e_{\psi}(\delta) d \delta, \quad e_{\psi}=\psi_{r e f}-\psi \\
& x_{z}=\int_{0}^{t} e_{z}(\delta) d \delta, \quad e_{z}=z_{r e f}-z_{c}
\end{aligned}
$$

Define the error signal $e=y-r$. The error signal $e$ can be rewritten in the matrix form as

$e=y-r=C x-I_{4} r$

Second, for penalizing the outputs $U_{1}, U_{2}, U_{3}, U_{4}$ of the system, the weight functions $W_{u_{i}}, i=1,2,3,4$ are added to the system as depicted in Fig (3). The system matrices of weight functions $W_{u_{i}}, i=1,2,3,4$ are $A_{u_{i}}, B_{u_{i}}, C_{u_{i}}$, and $D_{u_{i}}$.

Then, the dynamic of the all the weight functions $W_{u_{1}}$, $W_{u_{2}}, W_{u_{3}}$ and $W_{u_{4}}$ can be constituted as

$\left\{\begin{array}{l}\dot{x}_{u}=A_{u} x_{u}+B_{u} u \\ y_{u}=C_{u} x_{u}+D_{u} u\end{array}\right.$

where $x_{u}=\left[\begin{array}{llll}x_{u_{1}} & x_{u_{2}} & x_{u_{3}} & x_{u_{4}}\end{array}\right]^{T}$ is the state, $u=$ $\left[\begin{array}{llll}U_{1} & U_{2} & U_{3} & U_{4}\end{array}\right]^{T}$ represents the input, $y_{u}=$ $\left[\begin{array}{llll}z_{1} & z_{2} & z_{3} & z_{4}\end{array}\right]^{T}$ is the outputs of weight functions, and the system matrices of the weight function in (19) can be deducted as follows:

$\Delta_{u}=\left[\begin{array}{cccc}\Delta_{u_{1}} & 0 & 0 & 0 \\ 0 & \Delta_{u_{2}} & 0 & 0 \\ 0 & 0 & \Delta_{u_{3}} & 0 \\ 0 & 0 & 0 & \Delta_{u_{4}}\end{array}\right], \Delta \in\{A, B, C, D\}$

The augmented system with the new states, weight functions is depicted in Fig. 3.

Define $w=\left[\begin{array}{ll}r & d\end{array}\right]^{T}, z=\left[\begin{array}{ll}y_{u} & e\end{array}\right]^{T}$, and $\tilde{x}=$ $\left[\begin{array}{lll}x & x_{e} & x_{u}\end{array}\right]^{T}$ respectively as the exogenous input, exogenous output, and state of the augmented affine parameter-dependent. The affine parameter-dependent of the system differential equations in (8) with augmented states and weight functions can be regathered from (8), (12), (18), and (19) as follows:

$\left\{\begin{aligned} \dot{\tilde{x}} & =\sum_{i=1}^{16} \mu_{i}\left(\tilde{A}_{i} \tilde{x}+\tilde{B}_{1_{i}} w+\tilde{B}_{2} u\right) \\ z & =C_{1} \tilde{x}+D_{11} w+D_{12} u\end{aligned}\right.$ where

$$
\begin{aligned}
& \tilde{A}_{i}=\left[\begin{array}{ccc}
\bar{A}_{i} & 0 & 0 \\
-C & 0 & 0 \\
0 & 0 & A_{u}
\end{array}\right] ; \tilde{B}_{1_{i}}=\left[\begin{array}{cc}
0 & \bar{E}_{i} \\
-I_{4} & 0 \\
0 & 0
\end{array}\right] \\
& \tilde{B}_{2}=\left[\begin{array}{c}
\bar{B}_{i} \\
0 \\
B_{u}
\end{array}\right] ; C_{1}=\left[\begin{array}{ccc}
0 & 0 & C_{u} \\
C & 0 & 0
\end{array}\right] \\
& D_{11}=\left[\begin{array}{cc}
0 & 0 \\
-I_{4} & 0
\end{array}\right] ; D_{12}=\left[\begin{array}{c}
D_{u} \\
0
\end{array}\right]
\end{aligned}
$$

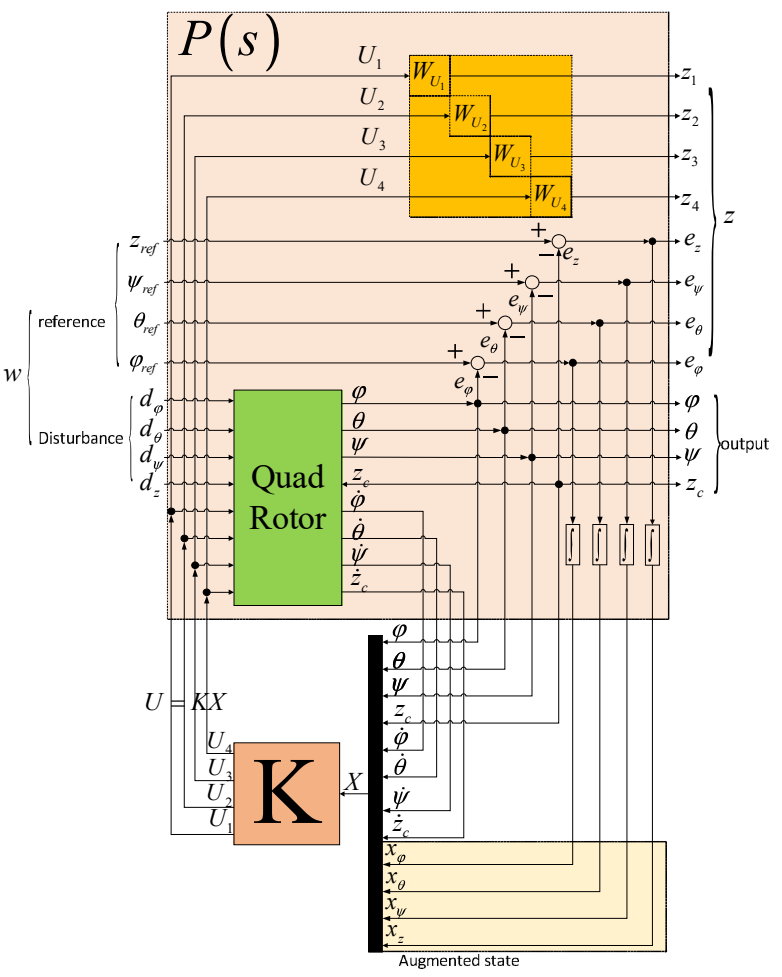

Fig. 3 Block diagram of the attitude robust controller with augmented states and weight functions

The aim now is to design the LPV $H_{\infty}$ optimal statefeedback controller of the form

$u(t)=\left(\sum_{i=1}^{16} \mu_{i} K_{i}\right) \tilde{x}(t)$

making the closed-loop system

$\dot{\tilde{x}}(t)=\sum_{i=1}^{16} \mu_{i}\left(\left(\tilde{A}_{i}+\tilde{B}_{2} K_{i}\right) \tilde{x}(t)+\tilde{B}_{1_{i}} w\right)$

robustly asymptotically stable.

Define the LMIs for $H_{\infty}$ optimal state-feedback controller for all TS sub model with common matrix $X$ and each $Y_{i}$ for each TS sub model based on Theorem 1 and 
poles location conditions in (15) as

$$
\begin{aligned}
& \underset{\gamma, X, Y_{1}, \ldots, Y_{16}}{\operatorname{minimize} \gamma} \\
& \text { subject to } \\
& X=X^{T} \succ 0 \\
& {\left[\begin{array}{ccc}
H e\left(\bar{A}_{i} X+\bar{B}_{2} Y_{i}\right) & (*)^{T} & (*)^{T} \\
\bar{B}_{1_{i}}^{T} & -\gamma I & (*)^{T} \\
\bar{C}_{1 i} X+D_{12} Y_{i} & \bar{D}_{11_{i}} & -\gamma I
\end{array}\right] \prec 0} \\
& \operatorname{He}\left(\bar{A}_{i} X+\bar{B}_{2} Y_{i}\right)+2 \alpha X \prec 0 ; \quad i=1 . .16
\end{aligned}
$$

By solving the LMIs in (23), the optimal $H_{\infty}$ state feedback controller with the smallest attenuation level $\gamma>0$ for the attitude/altitude sub system of the mass-varying quadcopter can be formulated as

$K(\rho)=\sum_{i=1}^{16} \mu_{i} Y_{i} X^{-1}$

\section{PRACTICAL CONTROLLER DESIGN}

We consider Takagi-Sugeno model where the mass varies in the interval interval $[\underline{m}, \bar{m}]$ with $\underline{m}=1.12(\mathrm{~kg})$ and $\bar{m}=2.0(\mathrm{~kg})$. The moments of inertia $I_{x}=I_{y}$ varies in the interval $\left[\underline{I_{x}}, \overline{I_{x}}\right]$ with $\underline{I_{x}}=0.0119\left(\mathrm{~kg} . \mathrm{m}^{2}\right)$ and $\overline{I_{x}}=0.0142\left(\mathrm{~kg} \cdot \mathrm{m}^{2}\right)$. The moments of inertia $I_{z}$ varies in the interval $\left[\underline{I_{z}}, \bar{I}_{z}\right]$ with $\underline{I_{z}}=0.0223\left(\mathrm{~kg} . \mathrm{m}^{2}\right)$ and $\overline{I_{z}}=0.0267\left(\mathrm{~kg} \cdot \mathrm{m}^{2}\right)$. The total residual angular speed $\Omega_{r}$ of motors varies in the interval $\left[\underline{\Omega_{r}}, \overline{\Omega_{r}}\right]$ with $\underline{\Omega_{r}}=$ $-1000\left[\mathrm{rad} \cdot \mathrm{s}^{-1}\right]$ and $\overline{\Omega_{r}}=1000\left[\mathrm{rad} \cdot \mathrm{s}^{-1}\right]$. The controller is designed using the procedure developed above.

The quadcopter parameters for simulation are listed in the following table (Fig. 1). Based on the quadcopter's

Table 1 Quadcopter parameters definition

\begin{tabular}{|l|l|l|l|}
\hline Par. & Name & Value & Unit \\
\hline$m$ & Quad. mass & 2.0 & $\mathrm{Kg}$ \\
\hline$l$ & Arm length & 0.23 & $\mathrm{~m}$ \\
\hline$I_{x}, I_{y}$ & Inertia vs $x, y$ & 0.0142 & $\mathrm{Kg} \cdot \mathrm{m}^{2}$ \\
\hline$I_{z}$ & Inertia vs $z$ & 0.0267 & $\mathrm{Kg} \cdot \mathrm{m}^{2}$ \\
\hline$J_{r}$ & Rotor inertia & $8.5 \times 10^{-4}$ & $\mathrm{Kg} \cdot \mathrm{m}^{2}$ \\
\hline$\omega_{i}$ & Rotor speed & {$[0,500]$} & $\mathrm{rad} / \mathrm{s}$ \\
\hline$\kappa_{i}$ & Rotor time const & 15 & $\mathrm{rad} / \mathrm{s}$ \\
\hline$g$ & Gravity accel. & 9.81 & $\mathrm{~m} / \mathrm{s}^{2}$ \\
\hline
\end{tabular}

parameters in Table 1, and the definition of varying parameters in subsections 2.3 , the ranges of varying parameters are shown in the Table 2.

\section{TESTING SCENARIO}

In simulations, the mass of the quadcopter is varying abruptly between $5 s$ and $25 s$ from $2(\mathrm{~kg})$ to $1.12(\mathrm{~kg})$. Along with the quadcopter's mass variation, the moments of inertia $I_{x}, I_{y}, I_{z}$ for $I_{x}=I_{y} \in\left[\begin{array}{ll}0.0119 & 0.0142\end{array}\right]$, and $I_{z} \in\left[\begin{array}{ll}0.0223 & 0.0267\end{array}\right]\left[\mathrm{kg} \cdot \mathrm{m}^{2}\right]$ also abruptly change as in Fig. 4. Fig. 5 shows the responses of $\varphi, \theta, \psi$ and $z$ when the reference signals are impulses and the disturbances $d_{\varphi}, d_{\theta}, d_{\psi}$, and $d_{z}$ are impulses. Fig. 6 shows the
Table 2 Variation ranges of varying parameters

\begin{tabular}{|c|c|c|}
\cline { 2 - 3 } \multicolumn{1}{c|}{} & $\underline{\rho}_{i}, i=1,2,3,4$ & $\bar{\rho}_{i}, i=1,2,3,4$ \\
\hline$\rho_{1}$ & 47.09580 & 84.0336 \\
\hline$\rho_{2}$ & 35.84230 & 44.8430 \\
\hline$\rho_{3}$ & 0.5000 & 0.89290 \\
\hline$\rho_{4}$ & -74.1176 & 74.1176 \\
\hline
\end{tabular}

responses of $\varphi, \theta, \psi$ and $z$ when the reference signals are random, the $z$ reference signal is impulse, and the disturbances $d_{\varphi}, d_{\theta}, d_{\psi}$, and $d_{z}$ are random. Fig. 7 shows the responses of $\varphi, \theta, \psi$ when the reference signals $\phi, \theta, \psi$ are, the $z$ reference signal is step, and the disturbances $d_{\varphi}$, $d_{\theta}, d_{\psi}$, and $d_{z}$ are also sine.

The simulation results suggest that the proposed controller works well for various reference signals (impulse, random, constant, and sine) and several types of disturbances (impulse, random, constant, and sine).

The same simulations for gradual variation of mass are also conducted, the reference paths are also wellfollowed.

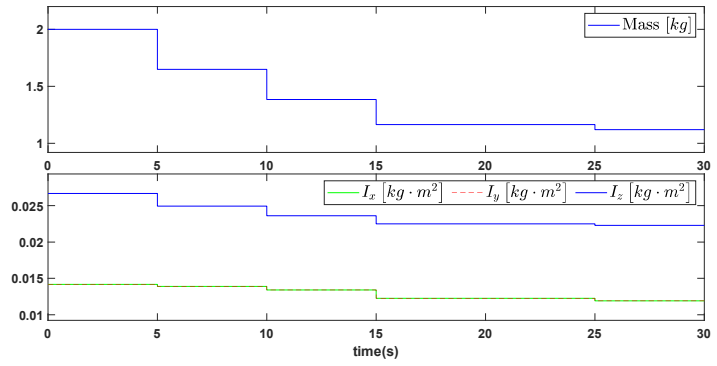

Fig. 4 Variations of Mass, $I_{x}, I_{y}$, and $I_{z}$
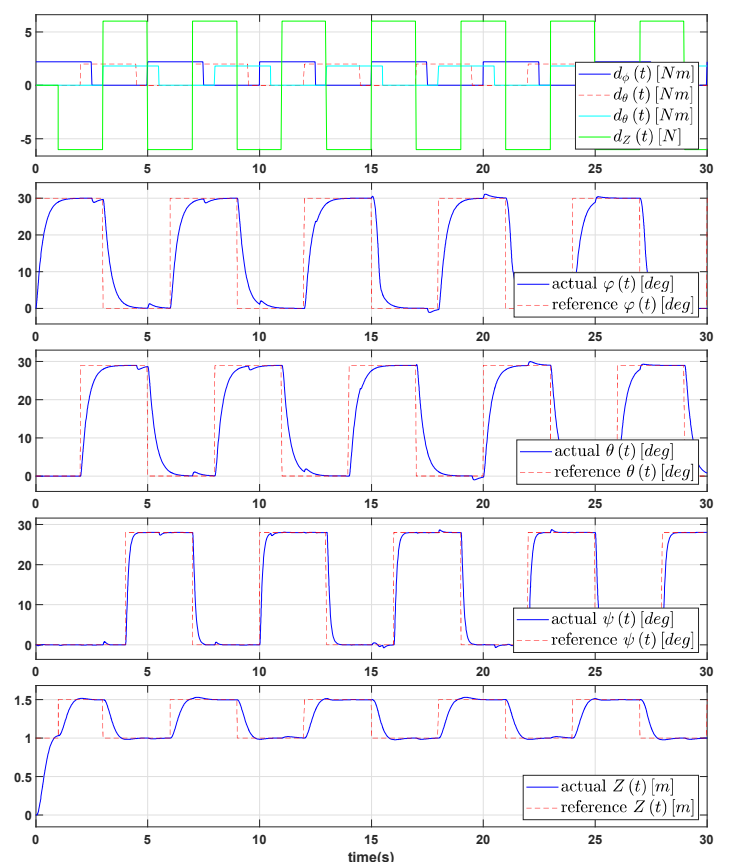

Fig. 5 Impulse references $\varphi, \theta, \psi$, impulse reference $z$, and impulse disturbances $d_{\varphi}, d_{\theta}, d_{\psi}, d_{z}$ 

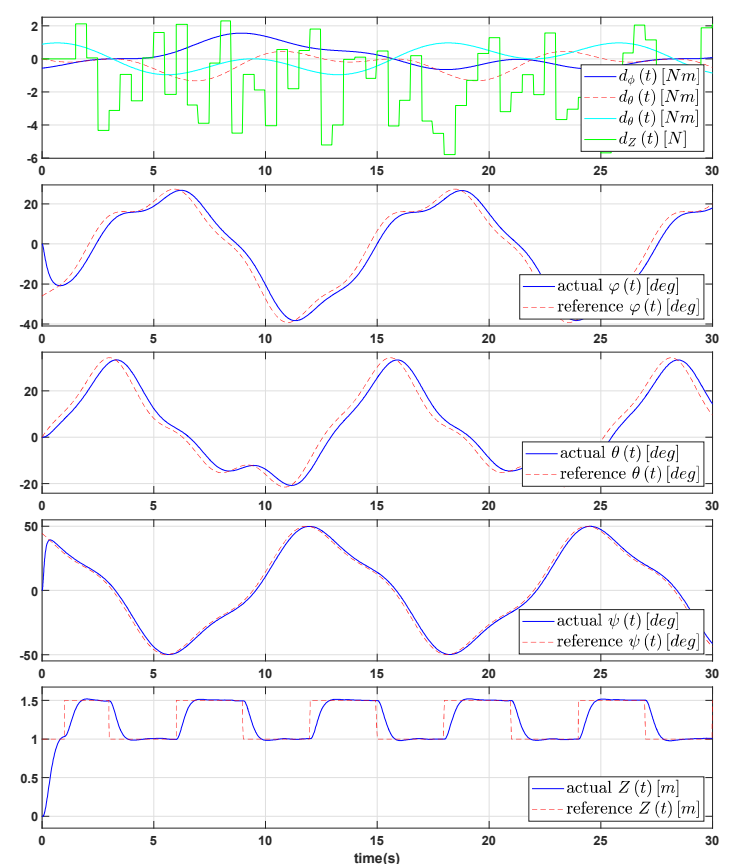

Fig. 6 Random references $\varphi, \theta, \psi$, impulse reference $z$, and random disturbances $d_{\varphi}, d_{\theta}, d_{\psi}, d_{z}$

\section{CONCLUSION}

This paper addresses the problem of attitude/altitude control of a quadcopter UAV. The focus is on handling mass, moments of inertia variation of the UAV according to the specific application of transporting different device types. By adding some additional state and weight functions, the linear parameter-dependent system is gathered. Thus the problem of reference tracking is formulated as $H_{\infty}$ state feedback. It is solved using the LMI conditions framework. The obtained controller is found to be able to follow the prescribed trajectory with a high level of performance even under disturbances and variations of dynamic parameters.

Future works concern the observer-based controller for a mass-varying quadcopter.

\section{REFERENCES}

[1] L. Besnarda, Y. B.Shtesselb, and BrianLandruma, "Quadrotor vehicle control via sliding mode controller driven by sliding mode disturbance observer," Journal of the Franklin Institute, vol. 349, no. 2, pp. 658-684, 2012.

[2] M. A. M. Basri, A. R. H. Rashid, and HusainKumeresan, "Feedback linearization vs. adaptive sliding mode control for a quadrotor helicopter," Sadhana, vol. 7, no. 3, pp. 419-428, 2009.

[3] S. Bouabdallah, A. Noth, and R. Siegwart, "Pid vs lq control techniques applied to an indoor micro quadrotor," Proceedings of 2004 IEEEIRS J Internationel Conference On Intelligent Robots and Systems September 28. October 2,2004, Sendal, Japan, pp. $2451-2456,2004$.
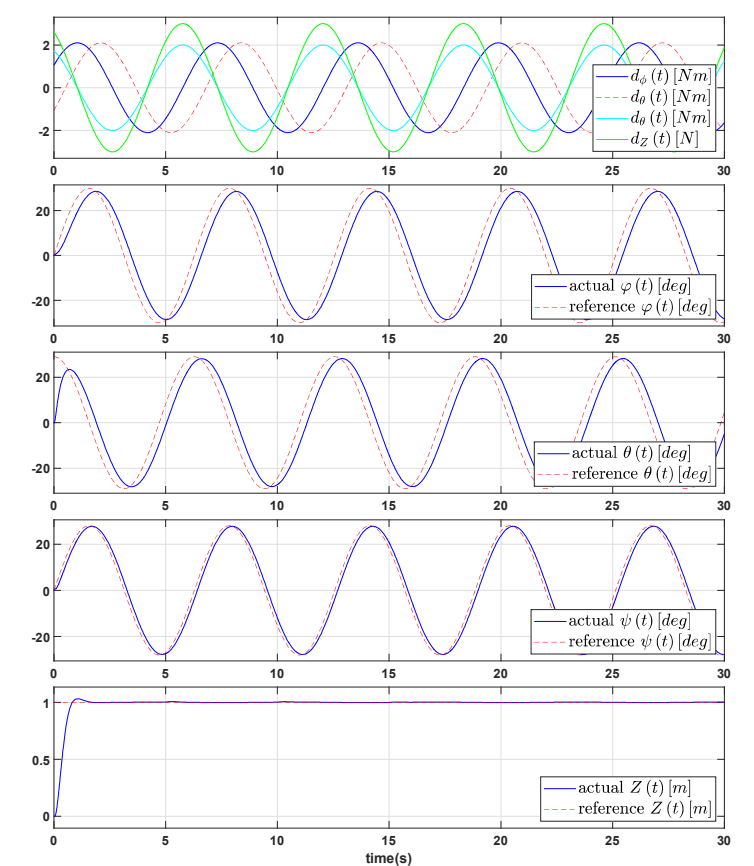

Fig. 7 Sine references $\varphi, \theta, \psi$, step reference $z$, and sine disturbances $d_{\varphi}, d_{\theta}, d_{\psi}, d_{z}$

[4] D. Lee, H. J. Kim, and S. Sastry, "Stabilization and trajectory tracking control for underactuated quadrotor helicopter subject to wind-gust disturbance," International Journal of Control, Automation and Systems, vol. 40, no. 5, pp. 1531-1553, 2009.

[5] D. Rotondo, F. Nejjari, and V. Puig, "Model reference quasi-lpv control of a quadrotor uav," 2014 IEEE Conference on Control Applications (CCA), vol. 8-10 Oct, Antibes, France, 2014.

[6] S. Bouabdallah, "Design and control of quadrotors with application to autonomous flying," 012007.

[7] S. Bouabdallah and R. Siegwart, "Backstepping and Sliding-mode Techniques Applied to an Indoor Micro Quadrotor," Intelligent Robots and Systems, 2007. IROS 2007. IEEE/RSJ International Conference, pp. 153-158, 2007.

[8] K. Tanaka and H. O. Wang, Fuzzy Control Systems Design and Analysis: A Linear Matrix Inequality Approach. Wiley Inc, New York, 2001.

[9] V. Wuest, V. Kumar, and G. Loianno, "Online estimation of geometric and inertia parameters for multirotor aerial vehicles," 2019 International Conference on Robotics and Automation (ICRA), vol. 2024 May 2019, Montreal, QC, Canada, Canada, pp. 1884 - 1890, 2019.

[10] B. Corentin, Linear Parameter-Varying and TimeDelay Systems. Analysis, Observation, Filtering and Control. Springer, 2015.

[11] C. Scherer, P. Gahinet, and M. Chilali, "Multiobjective output-feedback control via lmi optimization," IEEE Transactions on Automatic Control, vol. 42, no. 7, pp. 896-911, 1997. 